\title{
Low-grade endometrial stromal sarcoma with inferior vena cava tumor thrombus and intracardiac extension
}

\author{
Victor E Valdespino ${ }^{1 *}$, Hilda Mendoza Ramón ${ }^{1}$, German Maytorena Cordova² ${ }^{2}$ Victor Valdespino Gomez ${ }^{3}$ and Cynthia Lizarraga Mendez ${ }^{1}$ \\ ${ }^{1}$ Gynecology and Obstretrician Hospital, Mexican Institute Security Service "Luis Castelazo Ayala, Mexico \\ ${ }^{2}$ Pathological Anatomy, Mexican Institute Security Service "Luis Castelazo Ayala", Mexico \\ ${ }^{3}$ Care Attention Health department, University Metropolitan Autonomy, Mexico
}

\begin{abstract}
The endometrial stromal sarcoma is a rare neoplasm of the uterine origin. Intracardiac metastasis form the low-grade endometrial stromal sarcoma is an extremely rare event. A case of a patient who underwent surgical resection of metastatic tumors of the low-grade endometrial stromal sarcoma in the right atrium is described in the present report. Cardiac malignancies account for less than $1 \%$ of cardiac surgery and around $0.1 \%$ of cardiac echo graphic studies. The presence of metastatic tumor to the heart usually indicates widespread metastases. Lung carcinomas are the most commonly encountered tumor followed by breast and pancreas cancer and melanoma.

We show the case report of a woman with endometrial stromal sarcoma who developed a right heart failure, lower extremity edema, dyspnea and orthopnea after the initial step of comprehensive surgery and radiotherapy. Heart metastases was diagnosed by computed tomography and echocardiophy after a palliative metastasectomy; the patient received chemotherapy and hormotherapy without showing response, re-developing a heart metastasic tumor. Tumoral activity was the cause of her death.

It is very difficult to standardize care procedures for these patients due to the rarity of this condition. However, it is possible that aggressive therapy (surgery plus radiotherapy and chemotherapy) may lengthen patients survival and improve life quality.
\end{abstract}

\section{Introduction}

Endometrial sarcoma frequently spreads out to cervix, mucosa and myometrium of the lower uterine segment being lungs, liver and lymph nodes the common extra pelvic sites . However, endometrial sarcoma with cardiac metastasis is very uncommon.

Endometrial stroma sarcoma (ESS) represents $0.2-2 \%$ of all uterine malignancies. Based on the mitotic activity, a distinction is made between low and high-grade ESS and undifferentiated uterine sarcoma.

Endometrial stromal sarcoma are characterized by the presence of tumor thrombus is seen in renal cell and hepatocellular carcinoma, but is rarely reported in endometrial stromal sarcoma.

Low -grade endometrial stroma sarcoma is a malignant tumor composed of cells resembling stromal cells of proliferative-phase, infiltrative growth into the myometrium and /or lymph vascular spaces. It occurs over a wide age range with a mean of 52 years, but patients tend to be younger than those with other uterine sarcomas. Stage is the most important prognostic factor. Five-year disease specific survival for stage I and II is $90 \%$ compared to $50 \%$ for stage III and IV.

Patients typically present with abnormal uterine bleeding or abdominal pain. Less commonly they are asymptomatic, occasionally metastasis commonly ovary or lung. The uterus may be enlarged or there may be a pelvic mass. The frequency of adnexal involvement and lymph node metastasis is approximately $10 \%$ and up to $30 \%$ respectively. An association with prolonged estrogenic stimulation including tamoxifen, or history of pelvic radiation has been reported.
Endometrial stromal sarcomas are characterized by the presence of myometrial and or vascular invasion. They frequently are hormone receptor positive and positive for $\mathrm{CD} 10$ but not for desman or caldesmon.

The lung, breast, esophagus, malignant lymphoma, leukemia, and malignant melanoma are the most common tumors spreading to the heart. Cardiac metastases by the cancers occurring around infradiaphragmatic organs are less frequent. The incidence reported in literatures for the metastatic cardiac cancer is about $1.23 \%$. The cardiac metastasis is low because of continuous movement of myocardium, striated cardiac muscle, rapid circulation of blood and lymphatic flow away from the heart.

As already known, there are four pathways for cardiac metastasis. First, retrograde spread by lymph node, second, directly from adjacent viscera, blood circulation and through the vena cava.

Radiography is often the first imaging examination performed in the setting of new cardiac symptoms. However, the most common findings in cardiac metastatic disease are often nonspecific, including

Correspondence to: Victor E Valdespino, Gynecology and Obstretrician Hospital, Mexican Institute Security Service "Luis Castelazo Ayala, Mexico, Tel: 525573678619; E-mail: edvaldespinocg@yahoo.com

Key words: heart metastasis, endometrial stromal sarcoma

Received: August 09, 2017; Accepted: September 06, 2017; Published: September 09, 2017 
pleural effusions, an enlarged cardiac silhouette, or mediastinal lymphadenopathy. More suggestive findings of cardiac metastasis include a focal cardiac contour abnormality and radiographic evidence of a pericardial effusion. Any of these findings in a patient with a known malignancy should prompt further investigation, often beginning with echocardiography. Cardiac malignancies account for less $1 \%$ of cardiac surgery and about for $0.1 \%$ of cardiac echocardiogram studies. The presence of metastatic tumor to the heart usually indicates widespread metastases. Lung carcinomas are the most commonly encountered tumor followed by breast and pancreas cancer and melanoma for cardiac metastases.

When present, symptoms depend on the size and location of the metastasis. The most common symptoms attributable to cardiac metastasis are nonspecific and mimic other cardiac diseases such as myocardial ischemia, heart failure, or cardiac injury related to chemotherapy or radiation therapy. These symptoms include dyspnea, chest pain, palpitations, lower extremity edema, and new-onset atrial fibrillation [1-5].

\section{Case presentation}

We report a non-frequent case of endometrial stromal sarcoma low grade, extending into the superior vena cava and right atrium.

The patient was 45 year old, no chronic disease, and do not has health problems until present abnormal transvaginal bleed.

She was diagnosed like "uterine sarcoma" by endometrial biopsy. We performed total hysterectomy and salpingo-ophorectomy, and pelvic lymphadenectomy, the pathological stage was IB, with 24 nodes pelvic negatives. 8 .She was directed to radiotherapy to receive pelvic treatment and braquitherapy for $56 \mathrm{~Gy}$.

She was free of symptoms by 21 months, and she developed dyspnea, orthopnea and lower extremity edema. A plain chest radiograph indicated cardiomegaly. The patient developed clinical failure right heart failure.

A chest-abdominal computed tomography was performed revealing a filling defect initially thought to be thrombus in the IVC (inferior vein cava), extending into the right atrium; the tumor was enhanced heterogeneously on the CT scan. A transthoracic echocardiogram confirmed these findings.

Results of the laboratory test showed no significant abnormal value; even the hepatic and cardiac enzymes were within normal range.

Cardiothoracic services teams performed palliative resection of the tumor through open surgical excision of the mass. The total tumoral resection was $90 \%$.

After partial atrium and cava metastasectomy, the patient improved her life quality. Postoperatively, she felt more comfortable; as the chest pain and dyspnea had been resolved. She received chemotherapy, hormotherapy and observation by oncology and cardiac surgery then. She received 7 times epirrubicin with cardiac tumoral progression, so the chemotherapy drugs were changed to docetaxel and gemcitabine, showing poor response, and finally hormotherapy, with no response. The patient developed right heart failure again and died one year after palliated cardiac surgery and systemic treatment (Figures 1-6) [6-8].

\section{Discussion}

Endometrial stromal sarcoma constitutes approximately $0.2 \%$ to $1 \%$ of all uterine malignancies and approximately $10 \%$ of uterine

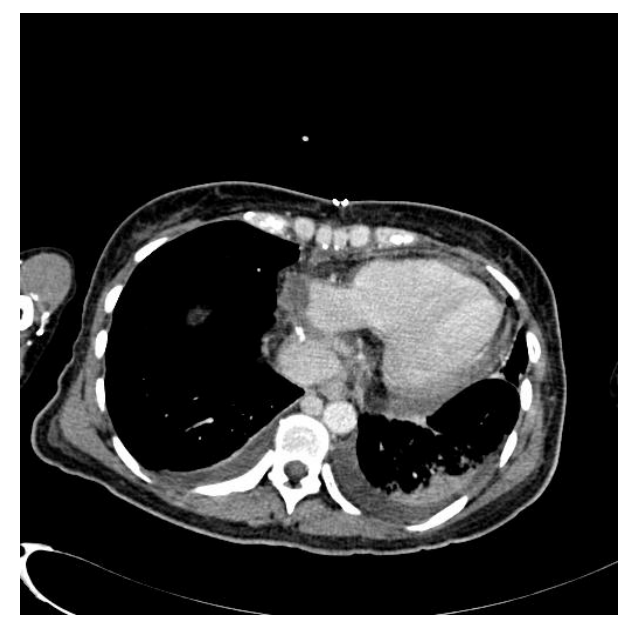

Figure 1. Computed tomo Gram (axial view) of the chest, shows a mass within the inferior vena cava, extending into the right atrium.

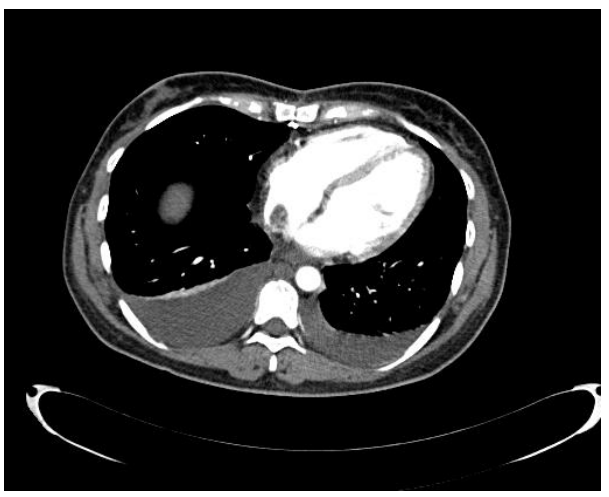

Figure 2. Computed tomographic revealed a filling defect, afther surgical resection

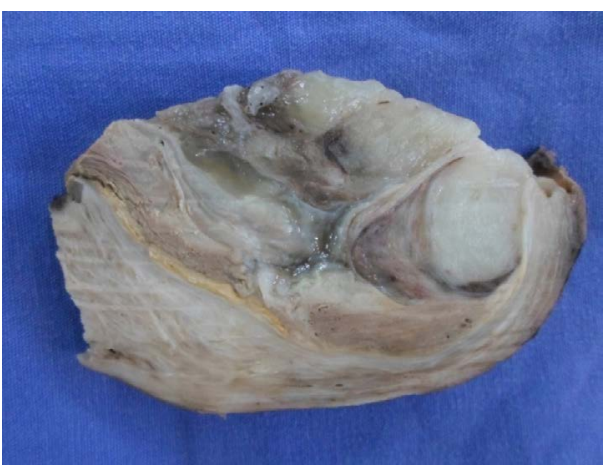

Figure 2. Computed tomographic revealed a filling defect, afther surgical resection

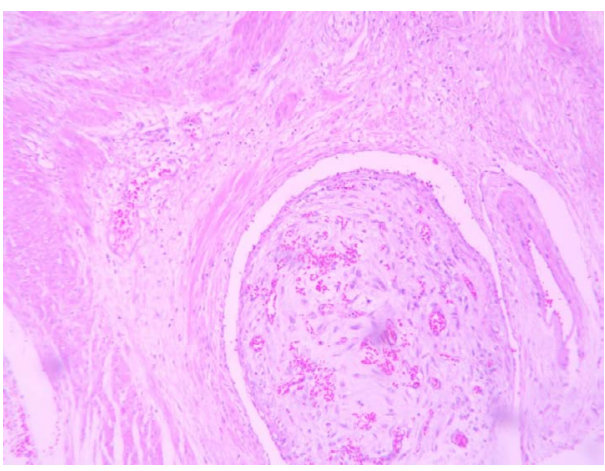

Figure 4. Microfotographty show miometrial wall and tumoral thrombus in vein 


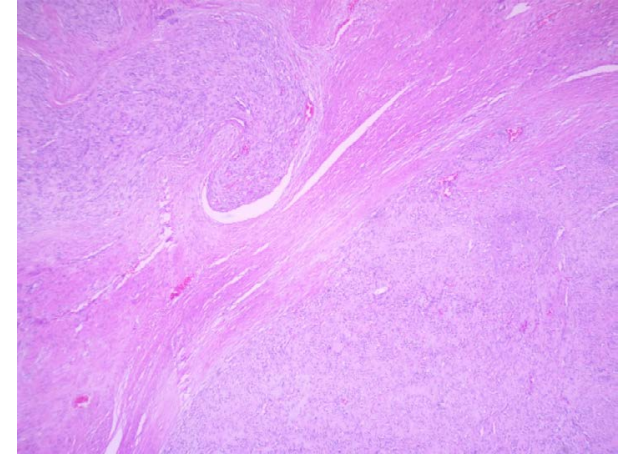

Figure 5. Microphography, uterine wall, fusocelullar tumor, typical aspect of "worm"

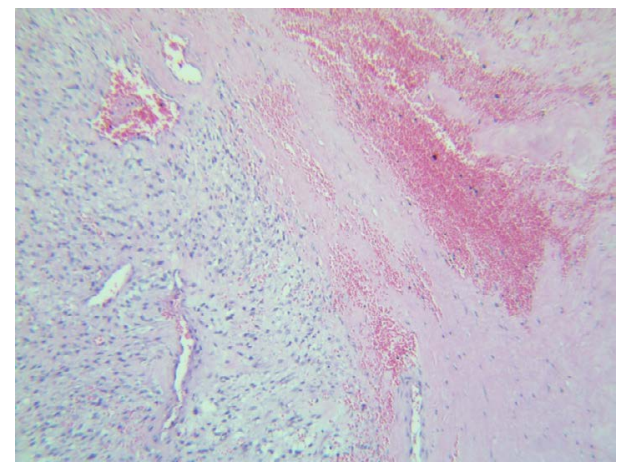

Figure 6. Tumor in right atrium, fusocelular tumor (endometrial stromal tumor) and blood tissue

sarcomas; its annual incidence is approximately 2 in 1,000,000 women. Its clinical recognition can be difficult, and it is often mistaken for a leiomyoma until a true diagnosis is made postoperatively. Histologic examiners frequently miss the diagnosis of early-stage, low-grade endometrial stromal sarcoma at a reported rate of up to $40 \%$, which results in therapeutic delay [9].

On the basis of the findings from imaging studies and the patient's medical history, we confirmed isolated metastasis of endometrial stromal sarcoma in the right atrium and inferior vena cava. Several reports have described cardiac tumor metastasis, but this presentation is very unusual. However, cardiac metastases usually invade the heart through the vascular system or by infiltrating the heart from neighboring organs. Cardiac metastases may be caused by the hematogenous spread of cancer cells. Diagnosis is difficult for patients who present with right cardiac cavities with tumor invasion. Yet, even with a diagnosis, effective treatment has not been well established. Presently, there is no standard treatment for metastatic endometrial stromal sarcoma with invasion into the right atrium. Palliative resection may be necessary owing to hemodynamic compromise, but the prognosis remains very poor. After or before partial surgical resection, we recommended chemotherapy, but this treatment was not useful; we were uncertain of the treatment effect, and the patient did not have good response. Until recently, only a few clinical studies of patients with endometrial stromal sarcoma extending into the heart have been published. Thus, more cases need to be accumulated and revised $[10,11]$.

Endometrial stromal sarcoma is classified as low-grade or highgrade, on the basis of mitotic activity. Low-grade tumors have fewer than 10 mitotic figures per high-power field, a 5-year survival rate of $80 \%$ to $100 \%$, and approximately a $50 \%$ rate of recurrence, typically after a long latency period. In one large series, the interval before recurrence varied from 3 months to 23 years, with a median interval of
3 years. Patients with high-grade endometrial stromal sarcoma have a 5 -year survival rate of $25-55 \%[3,9,11]$.

The present report describes an extraordinary rare case of isolated metastasis of endometrial stromal sarcoma in the right atrium. In similar cases, treatment should be individualized to the patient, and a multidisciplinary approach, including surgery and chemotherapy, should be used.

The heart is an uncommon site of metastasis due to relatively less vascularity of endocardium, repeated kneading movement of myocardium, rapid blood circulation and the lack of lymphatic communication of between the surrounding tissues. When the metastatic cancer is observed from the heart, the most common site is right side like in our case $[11,12]$.

Metastases in unusual locations are sometimes the first to be detected, and not uncommonly, single and prone to surgical resection.

Hepatocellular carcinoma and renal cell carcinoma are more frequent on the right side of the heart. The pericardium is the most commonly affected site by direct extension from mesothelioma, lung cancer, or breast cancer.

The most frequently reported sites of metastases of the low-grade subtype are the vagina, pelvis, and peritoneal cavity. Endometrial stromal sarcoma tends to spread throughout the lymph nodes and venous system but rarely involves the large vessels or the heart [13].

\section{Conclusion}

In a patient with clinical feature right heart, we have to consider heart metastases like a possibility of metastasis but first to consider others clinical conditions of heart failure because this metastases is extraordinary rare in current clinical practice; however this is possible. We need a high level of suspect if we want to found an infrequent metastases.

The treatment on a patient with heart metastasis most be surgical if possible, chemotherapy or hormotherapy did not have effectiveness in this rare metastases in this case.

\section{References}

1. Gabal S, Ashour Z, Hamada G, Aziz SA, Khairy H, et al. (2009) Low-grade endometrial stromal sarcoma with intravenous extension to the heart. Medscape J Med 11: 23.

2. Preti HA (2015) Points to Remember: Practical Pearls in Onco-Cardiology. Methodist DeBakey Cardiovascular Journal 11: 254.

3. World Health Organization Classification of Tumours (2014) WHO Classification of Tumours of Female Reproductive Organs.

4. Debourdeau P, Gligorov J, Teixeira L, Aletti M, Zammit C (2004) Malignant cardiac tumors. Bull Cancer 91 Suppl 3: 136-146. [Crossref]

5. Han GH, Kwon DY, Ulak R, Ki KD, Lee JM, et al. (2017) Right ventricular metastatic tumor from a primary carcinoma of uterine cervix: A cause of pulmonary embolism. Obstet Gynecol Sci 60: 129-132. [Crossref]

6. Meyers D, Nixon NA, Franko A, Ng D, Tam VC (2017) Tumour thrombus of the inferior vena cava extending into the right atrium in the setting of colon cancer. $B M J$ Case Rep 2017. [Crossref]

7. Bartosch C, Alfonso M, Pires-Luís AS, Galaghar A, Guimarães M, et al. (2016) Distant Metastases in Uterine Leiomyosarcomas: The Wide Variety of Body Sites and Time Intervals to Metastatic Relapse. Int J Gynecol Pathol.

8. American Joint Committee on Cancer (2017) Cancer Staging Manual. Eighth Edition, Springer.

9. Suzuki S, Yasuhara K, Koyano T, Obayashi T (2008) Tumor thrombectomy for endometrial stromal sarcoma extending into the inferior vena cava and the right atrium from the uterus. Kyobu Geka 61: 139-142. 
10. Renzulli P, Weimann R, Barras JP, Carrel TP, Candinas D (2009) Low-grade endometrial stromal sarcoma with inferior vena cava tumor thrombus and intracardiac extension: radical resection may improve recurrence-free survival. Surg Oncol 18: 57-64.

11. Yokoyama Y, Ono Y, Sakamoto T, Fukuda I, Mizunuma H (2004) Asymptomatic intracardiac metastasis from a low-grade endometrial stromal sarcoma with successful surgical resection. Gynecol Oncol 92: 999-1001.
12. Scher D, Nghiem W, Aziz S, Rahbar R, Banks W, et al. (2015) Endometrial Stromal Sarcoma Metastatic from the Uterus to the Inferior Vena Cava and Right Atrium. Texas Heart Institute Journal 42: 558-560.

13. Veroux P, Veroux M, Nicosia A, Bonanno MG, Tumminelli MG, et al. (2000) Thrombectomy of the inferior vena cava from recurrent low-grade endometrial stromal sarcoma: case report and review of the literature. J Surg Oncol 74: 45-48. [Crossref]

Copyright: ${ }^{0} 2017$ Valdespino VE. This is an open-access article distributed under the terms of the Creative Commons Attribution License, which permits unrestricted use, distribution, and reproduction in any medium, provided the original author and source are credited. 